Commentary on The Unfolding Case Formulation:

The Interplay of Description and Inference (Eells), and

Using Systematic Case Studies to Investigate Therapist Responsiveness:

Examples from a Case Series of PTSD Treatments (Edwards)

\title{
Pragmatic Clinical Research to Inform the Field of Men's Mental Health: A Commentary on and Extension of Papers by David Edwards and Tracy Eells
}

\section{CHRISTOPHER S. REIGELUTH ${ }^{\mathrm{a}, \mathrm{b}}$ \\ \& MICHAEL E. ADDIS ${ }^{\text {a }}$}

a Department of Psychology, Clark University

${ }^{\mathrm{b}}$ Correspondence concerning this article should be addressed to Christopher Reigeluth, Department of Psychology, Clark University, 950 Main Street, Worcester, M.A. 01610.

email: creigeluth@clarku.edu

\begin{abstract}
We consider the potential utility of the methods described by Eells (2010) and Edwards (2010) for guiding case conceptualization and treatment process in the area of men's mental health. As a relatively new field, men's mental health has been concerned with the clinical implications of understanding how masculine gender socialization influences the variety of ways different men experience, express, and respond to problems in their lives. Eells (2010) and Edwards (2010) have developed pragmatically driven research strategies that may help researchers and clinicians interested in working with men to (a) integrate gender in meaningful ways into case conceptualizations, and (b) develop effective ways of being therapeutically responsive to some of the specific challenges in working with more "traditional" men.
\end{abstract}

Key words: gender; gender socialization; social learning; masculinity; case formulation; case study; men's mental health

This commentary focuses on pragmatic approaches to case formulation and case study. Specifically, we consider the potential utility of methodologies described by Eells (2010) and Edwards (2010) by extending them to the relatively under-developed field of men's mental health. We begin by providing a brief overview of some of the current issues being considered in men's mental health. Next, we discuss two of these issues in greater depth: the need for gendered case formulations and the challenges underlying keeping some men in therapy. Our discussion about the pragmatic case study and case formulation centers on the ways that Eells' and Edwards' methodologies help address the above two issues. Lastly, we comment on the generalizability of these methods and provide some final thoughts on why Eells' and Edwards' approaches are especially well suited to inform men's mental health. 


\section{OVERVIEW OF MEN'S MENTAL HEALTH}

As a relatively new field, men's mental health primarily has been concerned with addressing different versions of the following question: how does masculine gender socialization influence the variety of ways different men experience, express, and respond to problems in their lives (Addis, 2008; Levant \& Pollack, 1995; Rochlen, 2005). Masculine gender socialization refers broadly to the ways that the gendered social meanings of masculinity (the pressures to be emotionally stoic and self-reliant, for example) come to shape men's thoughts, feelings, and behavior. This general framework for addressing men's mental health has clear clinical implications. For example, a fundamental assumption by the field is that social learning and gender norms influence men's help seeking behavior (Addis \& Mahalik, 2003). It has also been suggested that the social learning of masculine gender roles affects the way different men express disorders such as depression, anxiety, and substance abuse (e.g., Cochran \& Rabinowitz, 2000; Real, 1997). Below we consider four more specific clinically-relevant issues that provide a context for understanding the potential advantages of pragmatic methodologies such as those described by Eells and Edwards.

\section{1) Gender-Based Barriers to Help Seeking.}

Over the years, researchers have demonstrated that men who have been strongly influenced by traditional masculine norms are often averse to help seeking (Addis \& Mahalik, 2003; Berger et al., 2005; Good \& Wood, 1995; Smith et al., 2008; Steinfeldt et al., 2009). Theoretically, this can result either from individual men feeling that it is un-masculine to show vulnerability and weakness by going to therapy (e.g., admitting to "having a problem”), or from strict adherence to norms of emotional control. It can also be difficult for some men to relinquish their sense of personal control by placing themselves in the care of an authority figure. Finally, some men experience a great deal of anxiety when making themselves emotionally vulnerable. Such men often externalize their symptoms and shut down when prodded to explore their emotions. For all of these reasons, it is perhaps not surprising that men present for therapy at much lower rates than women (Addis \& Mahalik, 2003).

\section{2) The Challenge of Gender-Based Case Conceptualization.}

Another key issue for men's mental health is the challenge of knowing when it is necessary and appropriate to integrate gender into a case conceptualization. Gender socialization impacts different people in varying ways, and it is difficult to know when the influence of gender is clinically significant. When formulating a case, clinicians should consider the possible influence of gender in line with the client's values, personality traits, symptoms, and environmental contexts. Liu notes that while practitioners should not force discussion of masculine issues in therapy, they need to be aware of masculinity and consider its impact on the lives of the men they treat (2005). By considering gender at the start of the case conceptualization, clinicians will be more prepared for and responsive to the differing needs of their male clients. 


\section{3) Gender and the Expression of Psychopathology}

Some researchers and practitioners have raised the question of whether traditional diagnostic criteria adequately capture psychological distress and presenting symptoms in more traditional men (e.g., Addis, 2008; Rochlen, 2005). For example, it has been suggested that prototypic symptoms for diagnosing depression may not be present in some men and the disorder may be more likely to show up as anger, somatic symptoms, substance abuse, and so on (Addis, 2008). It is important for practitioners to be aware of the ways that gender norms can influence the expression of psychopathology. The underutilization of mental health services by men, as well as the under-diagnosis by practitioners, may be linked to an over-reliance on traditional diagnostic criteria.

\section{4) Lack of Empirical Evidence.}

The study of men's mental health is in need of innovative research approaches that will generate useful information about treatment with different types of men. To date, there are no large-scale randomized clinical trials (RCTs) that investigate the efficacy of specific treatments (Cochran, 2005). On the one hand, this is too bad because the field could benefit from findings generated by such studies. On the other hand, the good news is that, as a result, the field of men's mental health remains open to a wider range of research strategies that have the potential to inform clinical practice. The systematic case formulation and case study methodologies described by Eells and Edwards are good examples of the type of pragmatically driven research strategies that have the potential to do just this. Below we consider in more detail how both case formulation research and studies of therapist responsiveness can contribute to the developing knowledge in men's mental health.

\section{GENDER AND CASE FORMULATION}

As a key process that begins early in therapy, the case formulation lays the groundwork for understanding the client's history and presenting symptoms, and how to effectively strategize and implement treatment objectives (Eells et al., 1998). When working with male clients, one of the key questions for a therapist to consider is how to think about the role of gender in a client's life. There are several different ways to do this and how a practitioner conceptualizes gender can greatly influence the subsequent process of treatment. In short, each approach differs in the degree to which it conceptualizes gender through a lens of between-group, within-group, and intra-individual variability.

The first way to look at gender is through a lens of sex differences. This is perhaps the simplest and most intuitive way to think about what it means for a male client "to be a man." Clinicians might work from the assumption that gender represents categorical distinctions between males and females. In other words, a male client's thoughts, feelings, and behavior would be seen primarily in how they reflect fundamental differences from female clients. Clinicians working from a sex differences orientation would potentially integrate gender into a case formulation by discussing the role of sex differences in shaping that individual. Furthermore, by defining gender in line with sex differences, a clinician might conceptualize his 
or her client's gendered behaviors as more pre-determined and limited within that individual's male sex differences.

A second approach to integrating gender within a case formulation focuses on individual differences between men in the degree to which they have been affected by gendered social learning. Gendered social learning is the process by which men learn to behave in socially meaningful ways that mark them as conforming to societal expectations of masculinity. Some men have been strongly influenced by gendered social learning and consistently adhere to traditional and easily identifiable masculine norms. Other men are less affected and more visibly embrace non-traditional, non-gender specific behavioral norms. This approach emphasizes a male client's unique social learning contexts and the ways that he is individually different from other men. To integrate gender into the case formulation requires consideration of the potential impacts of social learning and environmental contexts on the client's personality traits, thoughts, and beliefs. In addition, a clinician might pose questions that take into account the client's unique life experience in relation to other men. In the case of some men, gendered social learning will influence if they approach therapy with openness or reluctance. It could also lead some men to externalize problems or face them directly. Lastly, gendered social learning could impact the types of therapeutic interventions a male client will consider.

A third approach to integrating gender focuses on the client's intra-individual variability and the ways that masculinity varies and shifts in different contexts of a man's life. In contrast to integrating gender into a case conceptualization by thinking about a male client in relation to other men, this approach considers a client's individual variability in response to the situations that he encounters. Tarrier (2002) speaks to the importance of considering social behavior and context in case formulation and identifies weak case formulations that focus on pure behavioral analysis. He provides specific examples of social factors, such as interpersonal environment and social supports, for the clinician to centrally consider. Similarly, a clinician might observe a client's gender-based behavior in terms of the influence of varying environmental contexts. Furthermore, a clinician who focuses on a client's intra-individual variability will most likely be cognizant of the potential impact of his or her own gender beliefs on the therapy session and the therapeutic alliance. From this perspective, masculinity is seen less as a property of individual men, and more as a potential characteristic of the therapeutic encounter that can emerge and dissolve depending on what is at stake in a particular interaction between therapist and client. Dynamics of interpersonal control and threats to self-esteem can be especially salient in this regard.

Through the Case Formulation Content Coding Method (CFCCM), Eells (2010) presents a system that has the potential to integrate gender into the conceptualization process at a variety of levels. In subcategory 21.2: Demographic/cultural factors (e.g., SES, gender), part of the Formulation/Inferred Information category, Eells creates a formulation category that mentions gender, but he could go further by coding distinct gender categories. There are several other stages of Eells' case formulation process, including Descriptive Information, Diagnostic Information, and Treatment Planning, which could also include gender specific codes. 
Of the possible ways to integrate gender within a case formulation, we will discuss a few examples that can be applied to Eells' coding system. In approaching gender from a sex differences/between group perspective, subcategory 22.2: Adaptive perceptions of or beliefs about others could be expanded to include the client's beliefs about being a man. For example, this could be a useful piece of information to include in a case formulation for a male client struggling to maintain intimate relationships. Men who consider emotional intimacy "feminine," and define masculinity as involving emotional control and rigid self-reliance, may have difficulty forming and maintaining close and emotionally vulnerable relationships.

In a second example that takes a between-groups approach, subcategory 21.3: Role conflict could be specified to include different types of role conflict including gender. For a male client involved in high-risk behavior (e.g., drinking excessively, driving dangerously, getting involved in fist fights), it would be important to question if the behaviors were socially learned and/or influenced by a desire to conform to masculine gender norms. It is important to raise this question because such an understanding could point to important social-environmental cues that make the maladaptive behavior more or less likely. Thus, Subcategory 21.3 could be expanded to include gender role conflicts.

A third example utilizes an intra-individual approach to gender. For example, in considering working with a hyper-masculine military veteran, who assumes an authoritative and dominant stance whenever in therapy, a therapist might initially use the case formulation to frame his or her client's masculine posturing. It would be useful to specifically question if the context of therapy was causing the client's domineering attitude and if there were specific times during the therapeutic exchange when his hyper-masculine behavior flared up. For understanding such a client's intra-individual variability through a gendered lens, subcategory 19.2: Problematic aspects of relatedness to others could be helpful. The code would need to be expanded to consider the ways that masculinity varies and shifts in relations with other people. By asking questions about gendered intra-individual variability during the case formulation phase, clinicians would be able to generate useful information, such as understanding the contexts and specific circumstances under which a male client's masculine gendered behavior becomes most problematic.

Eells also utilizes the CFCCM to compare novice, experienced, and expert case formulators in the content and quality of their coding. Expert case formulators typically generate a greater number of idea units in formulating their cases and spend more time analyzing the actual case material prior to drawing inferences. For expert therapists who work with men, it would be helpful to know if they integrate gender more often, and in what ways, than other therapists. Therapists who incorporate gender into their case formulations with men will be more prepared for possible challenges to treatment. Furthermore, Eells’ coding system has the potential to educate the field by highlighting the case formulations of therapists who successfully engage men in therapy and overcome gender-based barriers to help seeking. 


\section{THERAPIST RESPONSIVENESS AND KEEPING MEN IN TREATMENT}

Systematic case formulation that integrates gender is a step that can help clinicians address treatment barriers in some men. However, it is also important for clinicians to incorporate and be responsive to gender throughout therapy. Edwards (2010) sheds light on different types of therapist responsiveness through his systematic case study methodology. Edwards describes responsiveness as a therapist's ability to balance administering technical interventions with other factors such as a client's motivation and ability to sustain emotional focus and the importance of therapeutic alliance (2010). Edwards employs detailed and systematic case studies to shed light on and provide guidance for challenging cases. In thinking about applying Edward's case study model to working with difficult men, we focus on two key issues that therapist responsiveness helps to address: overcoming ambivalence about treatment in some male clients and ways to approach therapy with men who externalize symptoms. For both of these issues, systematic case studies can underscore effective and responsive approaches to overcoming gender-based barriers to treatment and building stronger therapeutic alliances.

A number of researchers and clinicians have suggested that some men can be especially ambivalent about the process of therapy (Addis \& Mahalik, 2003; Real, 1997; Rochlen, 2005; Steinfeldt, 2009). The act of seeing a therapist can destabilize traditional masculine ideals of stoicism, emotional control, and invincibility. As a result, some men resist encouragement from family members, and when finally convinced to attend therapy, they typically only last half as long as women (Kilmartin, 2007).

Understandably, overcoming a client's ambivalence about therapy is a major challenge and requires significant therapist responsiveness and flexibility. For example, in working with a male client who claims to have no problems, and is only seeking help because of pressure from a spouse, a clinician may take a less direct approach by building a strong alliance before launching into discussions of symptomatology. On the other hand, a therapist working with a client who sees exploring the depth of his emotions as a waste of time, and prefers a more solution-oriented approach, may be responsive to the client by working collaboratively to develop behavioral action plans. Of course, the same client may become ambivalent about treatment if he feels at all coerced, in which case responsiveness would involve the therapist recognizing the dynamics of interpersonal control and "toning down" the emphasis on change. In general, a crucial component of adequate responsiveness in working with male clients is a therapist's attention to the ways that gendered social learning has contributed to his or her client's ambivalence. Some therapeutic situations will demand that the therapist quickly adjust the emotional intensity in the room, others will require a swift and direct confrontation, and still others will call for using nonclinical language to communicate with more traditional men. In capturing these different types of responsiveness, Edwards' systematic case studies could be a useful resource in highlighting best practices for responsiveness and ways to effectively support the most challenging male clients.

Symptom externalization is a second issue that can challenge clinicians who work with male clients. Men who adhere to traditional masculine norms are often very reluctant to acknowledge that their problems involve internal emotional struggles, or that their own actions 
or beliefs may be contributing to their difficulties. In our own research group, we have come to call the extreme form of this the "asshole boss" syndrome (e.g., "I'm not depressed, I'm not angry, and there's nothing I can do about the situation; the problem is my asshole boss"). Responsiveness is a key factor for effectively engaging and keeping these men in therapy. A main challenge that clinicians can face is how and when to confront a client who externalizes symptoms. To be effectively responsive, the clinician needs to balance sensitivity to and validation of the client's construction of the problem with avoiding the temptation to collude. Collusion refers to a clinician's failure to understand and confront the male client's externalization, which can lead to a reinforcement of the externalized symptoms. On the other hand, if the therapist confronts a male client too abruptly, he or she risks eliciting unnecessary resistance and possibly losing the client.

\section{CONCLUSION}

As a relatively new field, men's mental health is in need of practical methodologies, such as Eells' (2010) and Edwards' (2010) innovative approaches to case formulation and case study. These methods can be relatively easily applied to clinical settings and utilized by practitioners. In addition, systematic case formulation and case study satisfy immediate needs in the field of men's mental health. For instance, Eells' methodology has the potential to integrate gender into case formulation and provide clinicians with more accurate conceptualizations of the ways that different men experience, express, and respond to problems in their lives. Edwards' systematic case studies serve a complementary role in providing the field with a method that captures different types of therapist responsiveness throughout the treatment process. As a result, clinicians working with difficult men can benefit from these two methodologies that span the treatment cycle from start to finish.

\section{REFERENCES}

Addis, M.E. (2008). Gender and depression in men. Clinical Psychology: Science and Practice, Vol. 15(3), 153-168.

Addis, M.E., \& Mahalik, J.R. (2003). Men, masculinity and the contexts of help seeking. American Psychologist, Vol. 58(1), 5-14.

Berger, J.M., Levant, R., McMillan, K.K., Kelleher, W., \& Sellers, A. (2005). Impact of gender role conflict, traditional masculinity ideology, alexithymia, and age on men's attitudes toward psychological help seeking. Psychology of Men and Masculinity, Vol. 6(1), 73-78.

Cochran, S.V. (2005). Evidence-based assessment with men. Journal of Clinical Psychology, Vol. 61(6), 649-660.

Cochran, S.V., \& Rabinowitz, F.E. (2000). Men and depression: Clinical and empirical perspectives: Practical resources for the mental health professional. San Diego, CA: Academic Press.

Edwards, D.J.A. (2010). Using systematic case studies to investigate therapist responsiveness: examples from a case series of PTSD treatments. Pragmatic Case Studies in Psychotherapy, 6(4), Article 3, 255-275. Available: http://hdl.rutgers.edu/1782.1/pcsp_journal 
Eells, T. D. (2010). The unfolding case formulation: The interplay of description and inference. Pragmatic Case Studies in Psychotherapy, 6(4), Article 2, 225-254. Available: http://hdl.rutgers.edu/1782.1/pcsp_journal

Eells, T.D., Kendjelic, E.M., \& Lucas, C.P. (1998). What's in a case formulation? Development and use of a content coding manual. Journal of Psychotherapy Practice and Research, Vol. 7(2), 144-153.

Good, G.E., \& Wood, P.K. (1995). Male gender role conflict, depression, and help seeking: Do college men face double jeopardy? Journal of Counseling and Development, 74, 70-75.

Kilmartin, C.T. (2007). The masculine self ( $3^{\text {rd }}$ ed.). Cornwall-on-Hudson, NY: Sloan Publishing.

Levant, R.F., \& Pollack, W.S. (Eds.). (1995). A new psychology of men. New York: Basic Books.

Liu, W.M. (2005). The study of men and masculinity as an important multicultural competency consideration. Journal of Clinical Psychology, Vol. 61(6), 685-697.

Real, T. (1997). I don't want to talk about it: Overcoming the legacy of male depression. New York: Fireside.

Rochlen, A.B. (2005). Men in (and out of) therapy: Central concepts, emerging directions, and remaining challenges. Journal of Clinical Psychology, Vol. 61(6), 627-631.

Smith, J.P., Tran, G.Q., \& Thompson, R.D. (2008). Can the theory of planned behavior help explain men's psychological help-seeking? Evidence for a mediation effect and clinical implications. (2008). Psychology of Men and Masculinity, Vol. 9(3), 179-192.

Steinfeldt, J.A., Steinfeldt, M.C., England, B., \& Speight, Q.L. (2009). Gender role conflict and stigma toward help-seeking among college football players. Psychology of Men and Masculinity, Vol.10(4), 261-272.

Tarrier, N., \& Calam, R. (2002). New developments in cognitive-behavioural case formulation. Epidemiological, systemic and social context: An integrative approach. Behavioural and Cognitive Psychotherapy, Vol. 30, 311-328. 